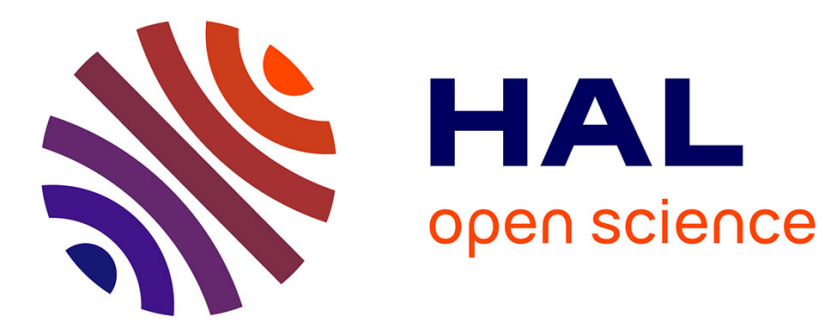

\title{
Effet de la date de plantation sur la croissance et le développement de plantules d'igname (Dioscorea alata L.) produites par culture in vitro
}

\author{
André Lacointe, Claude Zinsou, Camille Constant
}

\section{- To cite this version:}

André Lacointe, Claude Zinsou, Camille Constant. Effet de la date de plantation sur la croissance et le développement de plantules d'igname (Dioscorea alata L.) produites par culture in vitro. Agronomie, 1987, 7 (7), pp.475-481. hal-00885017

\section{HAL Id: hal-00885017 https://hal.science/hal-00885017}

Submitted on 1 Jan 1987

HAL is a multi-disciplinary open access archive for the deposit and dissemination of scientific research documents, whether they are published or not. The documents may come from teaching and research institutions in France or abroad, or from public or private research centers.
L'archive ouverte pluridisciplinaire HAL, est destinée au dépôt et à la diffusion de documents scientifiques de niveau recherche, publiés ou non, émanant des établissements d'enseignement et de recherche français ou étrangers, des laboratoires publics ou privés. 


\title{
Effet de la date de plantation sur la croissance et le développement de plantules d'igname (Dios- corea alata L.) produites par culture in vitro
}

\author{
André LACOINTE (*) \& Claude ZINSOU \\ avec la collaboration technique de Camille CONSTANT \\ I.N.R.A., Laboratoire de Physiologie et Biochimie végétales, Centre de Recherches des Antilles et de la Guyane,
} B.P. 1232, F 97184, Pointe-à-Pitre Cedex, Guadeloupe

RÉSUMÉ

\begin{abstract}
Après plantation à des dates échelonnées entre les mois de septembre et d'avril, de jeunes plantules de $D$. alata produites par culture in vitro, de morphologie comparable, ont été cultivées en conditions irriguées.

En plein champ, ces plantes diffèrent d'une plantation à l'autre par le développement et la croissance des parties aériennes (architecture et taille maximale) et par les rendements en poids de tubercules qui, pour les plantations des mois d'octobre à décembre, deviennent infimes par rapport aux plantations des mois de janvier à avril. Parallèlement, les variations dans les durées de végétation des plantes et de repos végétatif des tubercules-fils, suivant la date de plantation, sont telles que, dans tous les cas, les dates de germination des tubercules, situées sur 2 années successives, ne sont jamais très éloignées du mois d'avril, qui est l'époque de germination des tubercules en culture traditionnelle.

Ces résultats sont très proches de ceux généralement donnés par des plantations échelonnées de tubercules de $D$. alata présentant des signes plus ou moins nets de vieillissement. Ne pouvant, ici, les attribuer au tuberculemère, on en conclut que les problèmes rencontrés lors des tentatives de culture hors-saison de cette espèce sont liés surtout à sa sensibilité aux facteurs de l'environnement (thermo-photopériodisme).
\end{abstract}

Mots clés additionnels : Tubercule, tubérisation, culture hors-saison, agronomie tropicale.

Effect of planting date on growth and development of yam (Dioscorea alata L.) plantlets from in vitro culture.

D. alata plantlets from in vitro culture were planted at dates staggered between September and April and grown under irrigation conditions. Successive plantings did not initially differ in plantlet morphology, but further growth of both aerial parts (architecture and maximum size) and underground parts made them quite different from each other after a few months. Tuber yields of the October or December plantings were dramatically reduced compared to those of the January, March or April plantings. The duration of the leafy phase and of tuber dormancy also varied widely among plantings, so that tuber sprouting in all cases occurred around April, as observed in normal cultivation. These results were very close to those obtained from staggered plantings of more or less obviously aged $D$. alata tubers. Since they could not in this case be due to the mother-tuber, it was concluded that problems met when trying to cultivate the species out-of-season must mostly be related to its sensitivity to environmental conditions (thermo-photoperiodism).

Additional key words : Tuber, tuberization, out-of-season cultivation, tropical agronomy.

\section{INTRODUCTION}

En zone tropicale, si l'on excepte le facteur hydrique, le climat paraît a priori favorable toute l'année à la végétation. Les facteurs influant sur la productivité

(*) Adresse actuelle : I.N.R.A., Laboratoire de Bioclimatologie, Domaine de Crouelle, F 63039 Clermont-Ferrand Cedex. (température et durée d'insolation journalière) ne sont jamais limitants et restent relativement constants en regard des mêmes paramètres en zone tempérée. Pourtant, même en conditions irriguées, les essais de culture hors-saison ne réussissent pas pour certaines espèces. L'une des plus étudiées sous ce rapport est l'igname blanche, Dioscorea alata L., une plante tubérifère largement cultivée sous les Tropiques. 
En conditions naturelles, les tubercules de cette espèce sont en repos végétatif pendant environ 4 mois, de décembre à avril (hémisphère Nord). Le traitement des tubercules à l'éthylène chlorhydrine a permis à CAMPBELL et al. (1962) de hâter la germination et d'effectuer des plantations dès le mois de janvier : ni la durée de la phase végétative, ni les rendements, n'en furent affectés. Nous n'avons pas connaissance d'autre tentative de culture hâtée par réduction de la période de repos. En revanche, on relève dans la bibliographie plusieurs publications concernant des essais de culture retardée par allongement de la période de repos. Les moyens utilisés pour prolonger la conservation des tubercules hors-plantation sont très divers : traitement à l'hydrazide maléique (GoODING \& HOAD, 1967), ébourgeonnage (CLAIRON \& ZINSOU, 1980), stockage en chambre fraîche (ARNOLIN, 1981). Cependant, les résultats de ces différentes expérimentations sont voisins : plus tardive est la plantation entre juillet et novembre, plus courte est la phase végétative et plus réduits sont les rendements en tubercules.

Il semble donc que le comportement de $D$. alata soit directement dépendant de la date de plantation. Les variations des facteurs du milieu peuvent être à l'origine de cet effet. Cependant, les expérimentations dont on tirerait cette conclusion sont basées sur l'utilisation de tubercules conservés hors-plantation plus ou moins longtemps après la date normale de germination. Or, chez d'autres espèces comme la pomme de terre (PERENNEC \& MADEC, 1980), le vieillissement du tubercule entraîne une accélération de la tubérisation des germes aux dépens de la phase végétative. Le comportement observé chez l'igname peut donc avoir $a$ priori 2 origines distinctes : l'une interne (le vieillissement du tubercule-mère), l'autre externe (les variations des conditions du milieu).

Dans le but de dissocier ces 2 facteurs, nous avons entrepris des plantations échelonnées d'un matériel dépourvu d'effet « tubercule-mère ». D. alata ne produisant pas de graines, notre choix s'est porté sur des plants issus de culture in vitro (vitroplants).

Lors d'une précédente étude (LACOINTE \& ZINSOU, 1987), nous avons montré que le déroulement du cycle végétatif de ce matériel, après plantation fin avril (période de plantation des tubercules-semences traditionnels), est semblable à celui du matériel traditionnel, à part quelques particularités morphogénétiques qui apparentent les vitroplants aux jeunes semis d'espèces voisines.

Sans faire une analyse détaillée pour chacune d'elles, nous allons comparer le comportement des plantations effectuées hors-saison à celui de la plantation d'avril qui sera donc notre référence.

\section{MATÉRIEL ET MÉTHODES}

\section{A. Matériel végétal}

Les techniques de culture in vitro et d'adaptation des plantules aux conditions extérieures sont décrites par LACOINTE \& ZINSOU (1987). Les plantations ont été effectuées à raison de 60 à 120 individus tous les 40 jours entre le 17 septembre 1982 et le 22 avril 1983, en parcelles de 20 plantes tuteurées, les individus étant espacés de $1 \mathrm{~m}$ en tous sens.

\section{B. Cadre d'expérimentation}

Les cultures ont été réalisées en Guadeloupe $\left(16^{\circ}\right.$ Nord), Domaine Duclos (altitude : $120 \mathrm{~m}$ ), en conditions irriguées. L'amplitude thermique annuelle est voisine de l'amplitude thermique journalière : 5 à $7{ }^{\circ} \mathrm{C}$. Les températures les plus élevées sont situées en juillet-août, les plus faibles en janvier-février. La durée du jour varie entre $11 \mathrm{~h}$ et $13 \mathrm{~h}$.

\section{Mesures et prélèvements}

On effectue sur chaque plantation des prélèvements mensuels de 5 à 10 individus. On mesure le nombre et la longueur des tiges, les nombres de feuilles adultes et de feuilles en croissance (qui se distinguent des précédentes par leur teinte, leur aspect vernissé et leur orientation par rapport à la tige), la surface foliaire, le poids de matière sèche de la partie aérienne, le nombre de tubercules, et les poids de matière fraîche et de matière sèche de ces derniers. De plus, on suit individuellement la croissance et le développement des parties aériennes d'une dizaine de plantes de chacune des plantations des mois d'octobre, janvier et avril. (Dans la suite du texte, nous emploierons la forme elliptique « la plantation de (mars, juillet...) » pour « la plantation du mois de (mars, juillet...) ».) Ces 2 types de relevés, destructifs et non destructifs, sont effectués suivant le protocole de LACOINTE \& ZINSOU (1987).

\section{Statistiques}

Les comparaisons de plusieurs populations entre elles sont réalisées au moyen du test des rangs de Kruskal et Wallis (DAGNELIE, 1975).

\section{RÉSULTATS}

Initialement, la morphologie générale et le mode d'organogenèse des plantules mises au champ aux différentes dates sont toujours comparables à ceux décrits pour la plantation d'avril (LACOINTE \& ZINSOU, 1987). Nous ne parlerons ici que du comportement postérieur à la plantation.

\section{A. Croissance et développement de la partie aérienne}

Pour toutes les plantations, la partie aérienne se développe par émission de nouvelles tiges généralement groupées en un certain nombre de séries qui se succèdent au cours du temps. Les tiges des 2 ou 3 premières séries sont directement issues du collet (zone prétuberculaire) avec des vigueurs croissantes. Les 
séries suivantes comprennent principalement des rameaux axillaires, mais elles ne se manifestent pas sur toutes les plantations. L'architecture de la partie aérienne au maximum de son développement est donc très variable suivant la date de plantation (fig. 1, notamment $1 \mathrm{~b}$ ).
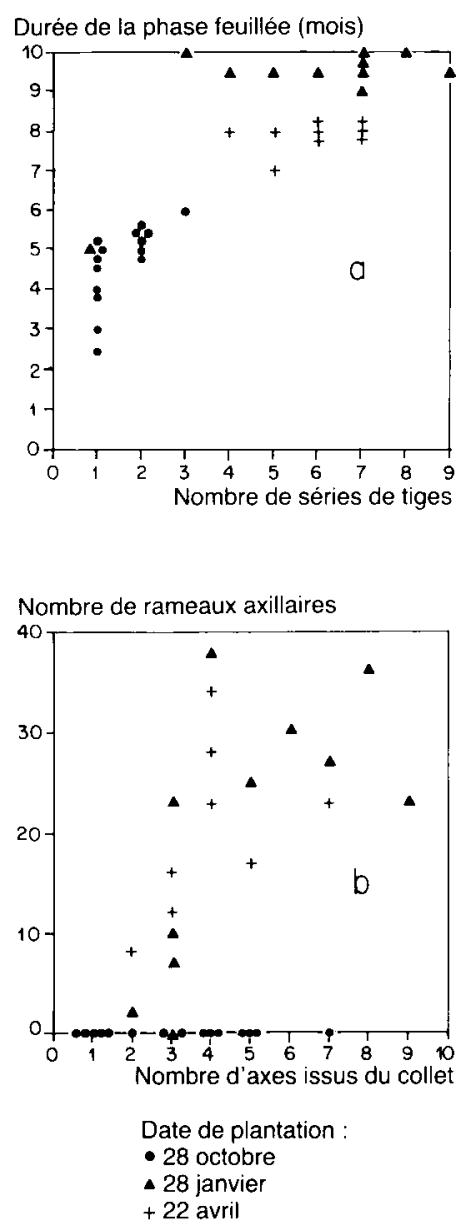

Figure 1

Construction de la partie aérienne des représentants de 3 plantations : chaque point représente l'ensemble des tiges émises (groupées en une ou plusieurs séries) par un individu durant la phase feuillée. a. Relation entre durée de la phase feuillée et nombre de séries de tiges; b. Répartition des tiges entre axes issus du collet et rameaux axillaires.

Structure of the aerial part of plants from three plantings : each point stands for the total set of stems formed (several at a time, making up one or several groups) by one individual during the leafy phase. a. Relation between leafy phase duration and number of stem groups. b. Proportion of stems from axillary shoots and from collar.

Quelle que soit la date de plantation, aucune série de tiges n'est émise de décembre à mars. Les rares feuilles en croissance présentes pendant ces 4 mois (fig. 2a) correspondent à des tiges émises, soit antérieurement à cette période, soit (pour les individus plantés pendant cette période) pendant la phase d'acclimatation en conditions semi-contrôlées (LACOINTE \& ZINSOU, 1987).

Comme la durée de vie d'une tige, après l'arrêt de sa croissance, excède rarement 3 mois, ces différences entre plantations affectant l'émission de nouvelles tiges entraînent de grandes disparités pour la durée de la phase feuillée comptée à partir de la mise au
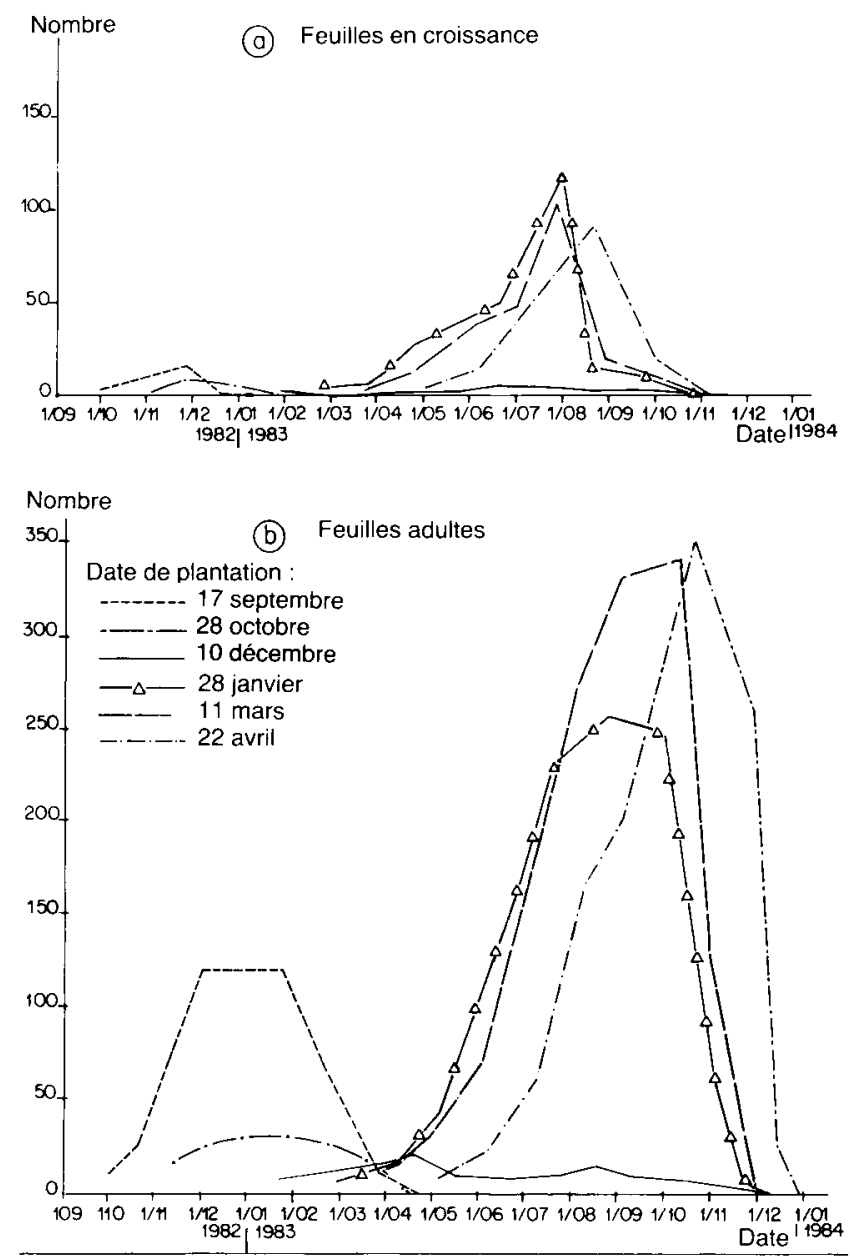

Figure 2

Evolution du nombre total de feuilles (moyennes de 5 à 10 individus). a. feuilles en croissance; b. feuilles adultes.

Variation in the total number of leaves (means of 5 to 10 individuals). a. growing leaves; b. full-grown leaves.

champ. Les différentes plantations se répartissent en 2 groupes (fig. 3 ) :

1) la totalité de celles de septembre et d'octobre, ainsi que respectivement 80 p. 100 et 10 p. 100 de cel-

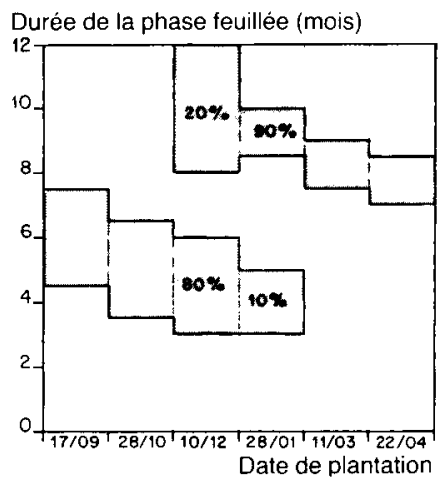

Figure 3

Durée de la phase feuillée : la zone hachurée regroupe 90 p. 100 des individus de la (sous-) population concernée. Les plantations de décembre et de janvier se répartissent en 2 sous-populations distinctes.

Duration of the leafy phase: the hatched area accounted for $90 \%$ of the individuals of the concerned (sub-) population. The December and January planting could be divided into 2 distinct subpopulations. 
TABLEAU 1

Valeurs maximales de quatre variables caractérisant la partie aérienne (moyenne de 5 à 10 individus).

Maximum values of four variables characteristic of the aerial part (means of 5-10 individuals).

\begin{tabular}{|c|c|c|c|c|c|c|c|}
\hline \multirow[t]{2}{*}{ Date de plantation } & \multirow[t]{2}{*}{$17 / 09$} & \multirow[t]{2}{*}{$28 / 10$} & \multicolumn{2}{|c|}{$10 / 12$} & \multirow{2}{*}{$\begin{array}{l}28 / 01 \\
(90 \%)\end{array}$} & \multirow[t]{2}{*}{$11 / 03$} & \multirow[t]{2}{*}{$22 / 04$} \\
\hline & & & $(80 \%)$ & $(20 \%)$ & & & \\
\hline NFA & 120 & 20 & 20 & 143 & 325 & 342 & 365 \\
\hline $\mathrm{SF}\left(\mathrm{cm}^{2}\right)$ & 2800 & 410 & 320 & 3000 & 12400 & 9700 & 13800 \\
\hline LCT $(\mathrm{cm})$ & 440 & 59 & 39 & 400 & 1640 & 1725 & 1470 \\
\hline PSA (g) & 17 & 2,0 & 2,2 & $?$ & 108 & 92 & 96 \\
\hline Groupe & 2 & 1 & 1 & 2 & 3 & 3 & 3 \\
\hline
\end{tabular}

NFA : nombre de feuilles; SF : surface foliaire cumulée ; LCT : longueur cumulée des tiges ; PSA : poids de matière sèche de la partie aérienne. Pour chacune de ces quatre variables, les différentes plantations (ou sous-populations) se répartissent en trois groupes homogènes significativement distincts les uns des autres $(\mathrm{P} \leqslant 0,05)$. Les quatre classements ainsi obtenus sont identiques (dernière ligne).

NFA : number of leaves ; SF : total leaf area ; LCT : total vine length ; PSA : aerial part dry matter weight. For each of these four variables, the different plantings (or subpopulations) were distributed in three homogeneous groups, significantly different from each other $(P \leqslant 0.05)$. The four classes derived from this are identical (lower line).

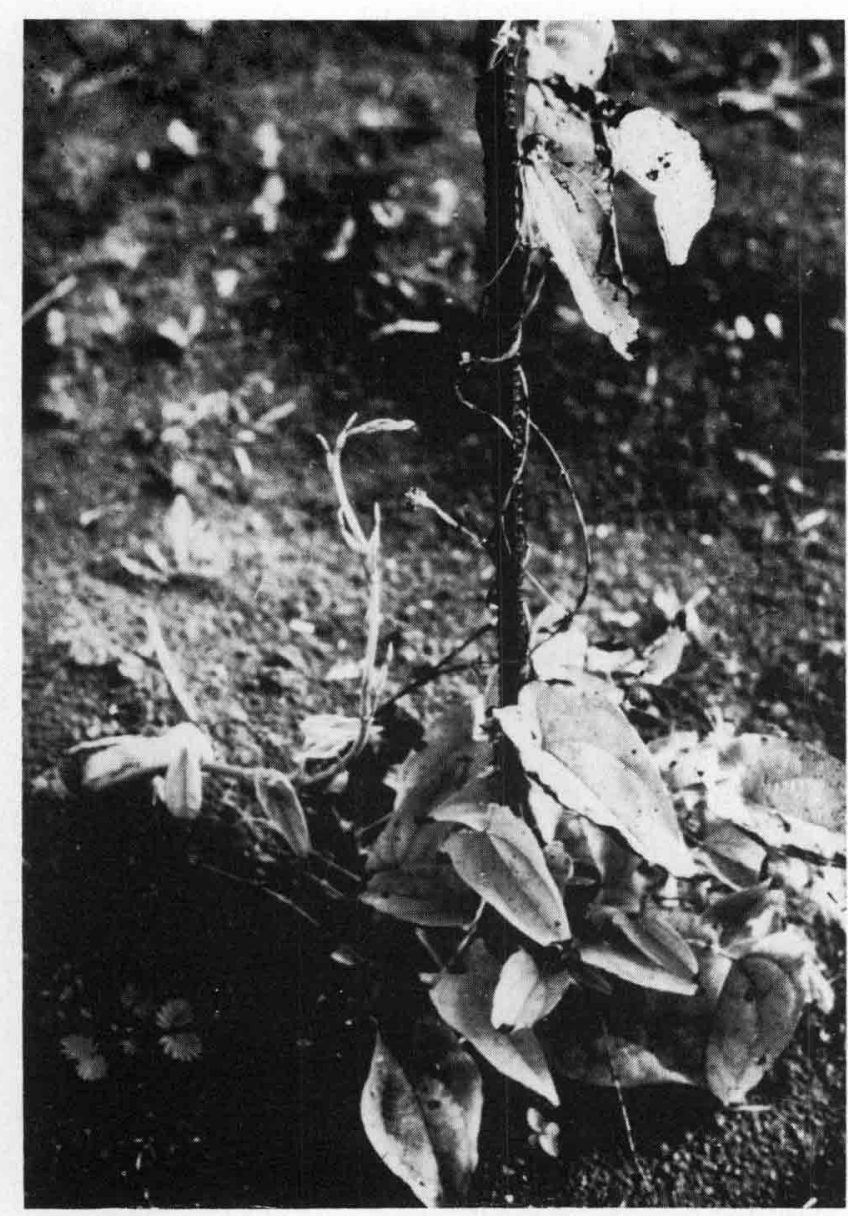

Figure 4

Reprise de croissance, au mois d'avril, de la partie aérienne d'un représentant de la plantation du 10 décembre. A gauche, jeune pousse vigoureuse émise par la zone prétuberculaire; à droite, lianes âgées enroulées autour du tuteur.

Growth resumption in April of the aerial part of an individual from the December planting. Left : strong young shoot emerging from the primary nodal complex region (head); right: old vines rolled around the stake. les de décembre et de janvier (voir aussi fig. 1a). Les parties aériennes de ces plantes ont disparu avant le mois d'avril et leur volume maximum est d'autant plus réduit que la plantation a été plus tardive (fig. 2 et tabl. 1) ;

2) la totalité de celles de mars et d'avril, ainsi que le restant de celles de décembre $(20$ p. 100) et de janvier ( 90 p. 100). Au mois d'avril, ces 2 dernières souspopulations ont émis des axes vigoureux au niveau du collet (fig. 4), leur partie aérienne finissant par atteindre une taille maximum comparable à celle des plantations ultérieures ou au moins à celle de la plantation de septembre.

Seules les plantations de janvier (sous-population 90 p. 100), mars et avril comptent quelques individus fleuris (environ 1 sur 5).

\section{B. Croissance et développement des tubercules}

Le poids des tubercules à la récolte varie de façon importante suivant la date de plantation (tabl. 2). Les différentes plantations se classent entre elles, suivant ce critère de rendement, dans le même ordre que suivant les critères du développement maximal aérien (tabl. 1). Ce n'est pas surprenant, le développement aérien étant évidemment, via la capacité photosynthétique, un facteur limitant pour le développement d'un organe de réserve.

Cependant, la croissance de la partie souterraine est affectée aussi par d'autres facteurs, comme l'indique une étude cinétique (fig. 5) :

1) les tubercules atteignent $10 \mathrm{~g}$ de matière fraîche par plante en 2 mois après plantation en octobre, contre 3,5 mois après plantation en mars ou en avril, bien que la partie aérienne, à 2 mois, soit plus développée dans le second cas que dans le premier (fig. 2a);

2) pour les plantes dont la partie aérienne est vivante en avril, la croissance des tubercules entre le 
TABLEAU 2

Rendement par plante à la récolte (poids de matière fraîche des tubercules). Le classement des différentes plantations suivant ce critère donne trois groupes homogènes et distincts les uns des autres $(P \leqslant 0,05)$.

Yield per plant at harvest (tuber fresh matter weight). Classing according to this criterion led to three homogeneous groups, different from each other $(P \leqslant 0.05)$.

\begin{tabular}{|c|c|c|c|c|c|c|c|}
\hline \multirow[t]{2}{*}{ Date de plantation } & \multirow[t]{2}{*}{$27 / 09$} & \multirow[t]{2}{*}{$28 / 10$} & \multicolumn{2}{|c|}{$10 / 12$} & \multirow{2}{*}{$\begin{array}{l}28 / 01 \\
(90 \%)\end{array}$} & \multirow[t]{2}{*}{$11 / 03$} & \multirow[t]{2}{*}{$22 / 04$} \\
\hline & & & $(80 \%)$ & $(20 \%)$ & & & \\
\hline PFT (g) & 380 & 63 & 17 & 555 & 670 & 850 & 760 \\
\hline Groupe & 2 & 1 & 1 & 2 & 3 & 3 & 3 \\
\hline
\end{tabular}

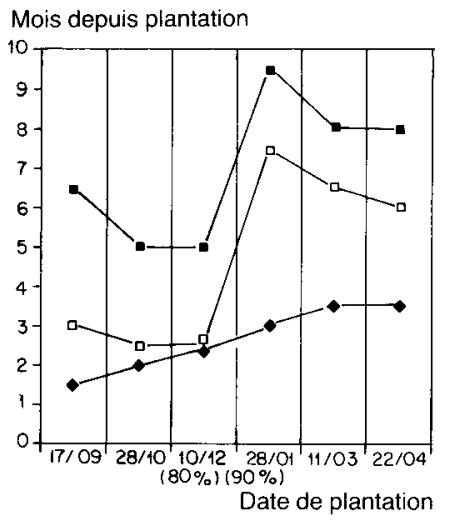

Figure 5

Trois paramètres caractérisant la cinétique de croissance des tubercules. Chaque courbe indique le délai entre la plantation et un stade défini par le poids de matière fraîche des tubercules:

poids maximum (= durée de la phase feuillée)

$\square-\square$ demi-poids maximum

- poids $=10 \mathrm{~g}$.

Three parameters characterizing the dynamics of tuber growth. Each curve shows the time (months) elapsed from planting to a stage defined by tuber fresh matter weight :

$\square$ maximum weight $(=$ duration of leafy phase $)$

$\downarrow$ weight $=10 \mathrm{~g}$.

stade $10 \mathrm{~g}$ et le stade demi-poids maximum (environ $350 \mathrm{~g}$ ) est d'autant plus lente que la plantation est plus précoce. Certains exemplaires des plantations de décembre et de janvier présentent même des signes de croissance en 2 phases nettement disjointes (fig. 6).

Ces observations peuvent se résumer ainsi : indépendamment de la date de plantation, la croissance du tubercule est rapide pendant les mois d'octobre à février ; elle est lente ou nulle pendant les mois de mars à juillet. La saison au cours de laquelle la croissance souterraine est intense correspond donc (approximativement) à la saison au cours de laquelle la croissance aérienne est faible ou nulle, et inversement. La succession de ces deux saisons apparaît sur les courbes représentant le rapport pondéral : partie aérienne/tubercules (fig. 7), sous forme de portions croissantes et décroissantes.

Ainsi, le grossissement des tubercules des plantations de septembre et d'octobre (respectivement 127 et $22 \mathrm{~g}$ de matière sèche à la récolte) est relativement moins affecté que celui de la partie aérienne ( 17 et $2 \mathrm{~g}$ de matière sèche au maximum), par rapport aux plantations de mars et d'avril (respectivement 280 et $251 \mathrm{~g}$

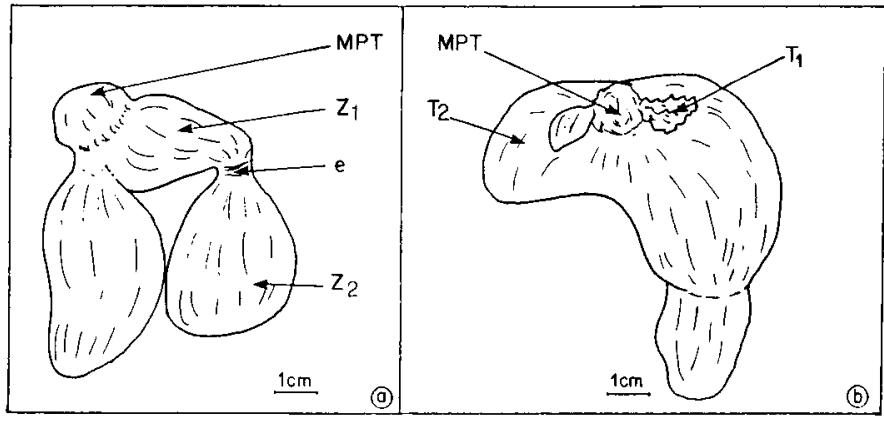

Figure 6

Tubercules présentant des signes de croissance en 2 phases (plantation : décembre 1983 ; récolte : décembre 1984, à la chute du système aérien).

MPT : zone prétuberculaire (« tête »)

a $\quad$ z : partie formée durant la $1^{\text {re }}$ phase

$e$ : étranglement séparant les 2 phases

$z 2$ : partie formée durant la $2^{e}$ phase

b TI: tubercule de "Ire génération 》, vide à la récolte T2: tubercule de " $2^{e}$ génération 》.

Tubers showing indications of growth in two phases (planting date December 1983; harvesting date : December 1984, at leaf fall) $M P T$ : primary nodal complex region (" head")

a $\quad$ z1: part grown during the lst phase $e$ : narrowing at the separation between the two phases z2: part grown during the 2nd phase

b $T 1:$ " lst generation " tuber, empty at harvest T2: «2nd generation 》 tuber.

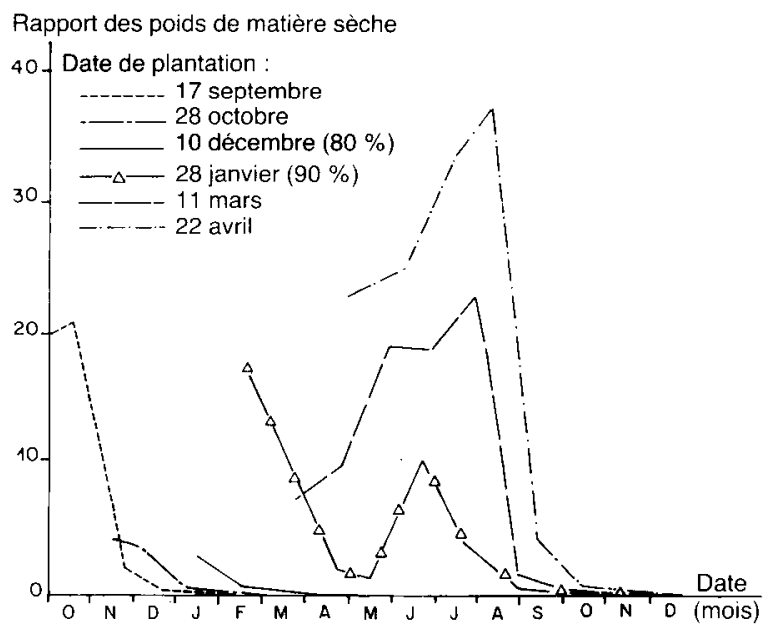

Figure 7

Evolution du rapport des poids de matière sèche $\left(\frac{\text { Partie aérienne }}{\text { Tubercule }}\right)$

Variation in $\frac{\text { aerial part }}{\text { tuber }}$ dry matter weight ratio. 
pour les tubercules, et 92 et $96 \mathrm{~g}$ pour la partie aérienne).

\section{La phase de repos végétatif}

La variation de la durée de la phase feuillée en fonction de la date de plantation (fig. 3) se traduit par le regroupement des dates moyennes d'entrée en repos végétatif des différentes plantations (fig. 8a) sur 2 saisons distantes de 7 mois :

1) mars à mai (plantations de septembre et d'octobre, ainsi que 80 p. 100 et 10 p. 100 respectivement de celles de décembre et janvier) ;

2) novembre-décembre (plantations de mars et d'avril, ainsi que le restant des plantations de décembre et janvier).

L'écart entre les dates moyennes de germination de ces 2 groupes de plantations est de 12 mois avec dans chaque groupe une période de germination s'échelonnant sur 2 mois, exception faite de la plantation de décembre (fig. 8b) caractérisée aussi par une forte hétérogénéité au niveau des plantes. Les écarts entre les dates de germination sont plus importants que ceux entre les dates de plantation parce que la durée $\mathrm{du}$ repos végétatif varie beaucoup en fonction de la date de plantation : de 5 mois pour la plantation de mars, à moins d'un mois (voire quelques jours dans certains cas) pour celle de septembre.

Quant aux cas de reprise de la croissance de la partie aérienne après un arrêt complet de plusieurs mois au cours desquels la plante avait conservé une partie de son feuillage (fig. 4), ils peuvent être considérés comme des cas de recouvrement de deux cycles végétatifs, la germination du tubercule se produisant avant la chute du feuillage. En effet les nouvelles pousses sont morphologiquement semblables à celles issues d'un tubercule en germination, et les émissions des deux types de pousses sont contemporaines (avril). De plus, la partie souterraine de certains exemplaires des 2 plantations concernées présente 2 générations successives de tubercules dont la $1^{\text {re }}$ est «vidée » (fig. 6b) : les réserves ont probablement servi à la reprise de la croissance aérienne, comme pour une germination.

\section{DISCUSSION ET CONCLUSION}

Le comportement des vitroplants de $D$. alata $\mathrm{cv}$. "Lupias " varie suivant la date de plantation. Cet effet se traduit essentiellement par :

1) une limitation importante de la croissance et du développement de la partie aérienne et, dans une moindre mesure, de la partie souterraine, si la plantation est située entre les mois de septembre et décembre. La diminution de rendement en tubercules, significative dès la plantation de septembre, devient désastreuse pour celles d'octobre et décembre ;

2) un certain regroupement des dates de disparition de l'appareil aérien, et un regroupement encore plus net, sur une période assez précise de l'année, des dates de germination des tubercules. Un décalage du cycle végétatif, par rapport au cycle normal sous les conditions naturelles, peut être ainsi annulé dès l'année suivante.

Il est donc clair que les plantations échelonnées de vitroplants de $D$. alata $\mathrm{cv}$. "Lupias ", du moins dans nos conditions de culture, ne permettent pas une production satisfaisante de tubercules à toute époque de l'année.

Ce résultat est semblable à ceux obtenus après plantation de tubercules-semences conservés quelques mois après la date normale de germination (cf. Introduction). Les comportements des deux types de matériel sont également voisins si la plantation est encore plus tardive : après plantation en décembre ou en janvier

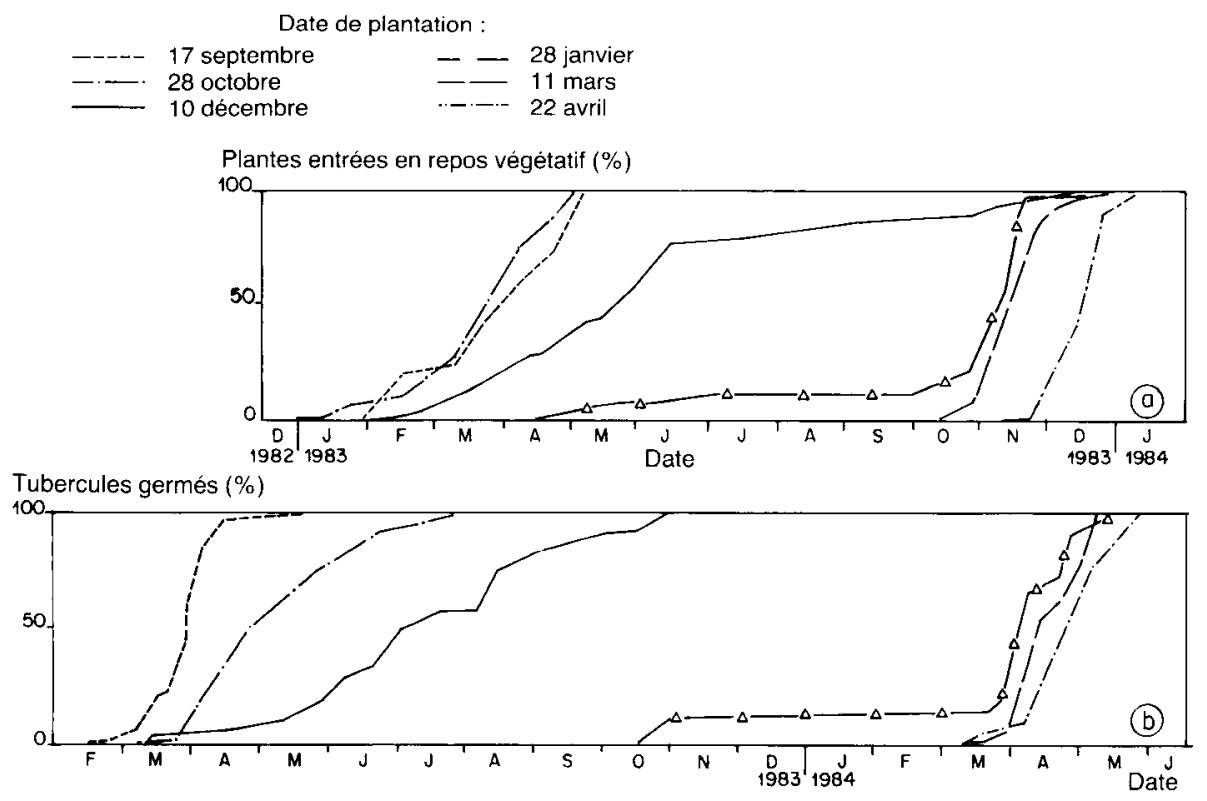

Figure 8

Repos végétatif du tubercule : cinétiques d'entrée en repos végétatif (a) et de germination (b).

Tuber dormancy : time-course of vine dying-down (a) and of tuber sprouting (b). 
de bulbilles de $D$. alata $\mathrm{cv}$. "Tahiti » stockées durant un an, la durée de la phase feuillée varie de façon " anarchique " entre 4 et 12 mois (ARNOLIN, 1981). Des tubercules plantés à partir de février, après plus d'un an de stockage, produisent des plantes vigoureuses à cycle végétatif normal (ARNOLIN, 1981; MATHURIN, 1982).

Comme nos vitroplants ne proviennent pas de tubercules-mères et présentent des caractères juvéniles, nous interprétons leur comportement comme une réponse aux variations saisonnières des facteurs de l'environnement. On peut penser que les résultats des expérimentations basées sur l'utilisation de tuberculessemences s'expliquent également ainsi dans une large mesure. Cela n'exclut pas cependant la possibilité d'un effet supplémentaire lié au vieillissement du tubercule, dont la manifestation peut d'ailleurs parfois n'être que transitoire, masquée par la réaction de la plante aux facteurs du milieu, comme le suggèrent les résultats de MATHURIN (1982).

Cet effet des facteurs externes paraît s'exercer principalement par le contrôle de la balance des croissances, jamais intenses simultanément, des deux « compartiments » que constituent respectivement la partie aérienne et les tubercules : la croissance de la première est prépondérante de mars à juillet et celle des seconds, d'octobre à février. Une telle incompatibilité entre croissance de la partie aérienne et tubérisation rappelle la physiologie des plantes tubérifères de la zone tempérée, comme la pomme de terre ou le topinambour (JOLIVET, 1969). Cependant, alors que cette incompatibilité est généralement très stricte en zone tempérée, elle n'est que relative dans le cas des ignames tropicales.

Quels sont les facteurs climatiques impliqués?
Ayant conduit nos expérimentations en conditions irriguées, nous pensons que le facteur hydrique (du moins au niveau du sol) n'est pas déterminant, bien qu'il soit de loin le plus limitant et que le cycle végétatif de l'igname en conditions naturelles soit remarquablement adapté à ses grandes variations saisonnières.

Ce sont donc probablement les variations, même de bien plus faible amplitude, d'autres paramètres tels que la photopériode et/ou la thermopériode, dont l'influence sur la tubérisation est bien connue en zone tempérée, qu'il faut mettre en cause. Nos expérimentations ne permettent pas de préciser davantage, mais celles de MIGINIAC (1980), effectuées à température constante $\left(27^{\circ} \mathrm{C}\right)$ sous diverses durées de photophase (non rencontrées dans la nature), laissent penser que l'influence de la photopériode est déterminante.

La saison favorisant le développement de la partie aérienne correspond alors aux conditions de « jours longs", et celle d'effet inverse aux conditions de « jours courts », conclusion similaire à celle qu'avait tirée NJOKU (1963) de l'étude du développement d'un autre matériel végétal (boutures de $D$. spp.).

Reçu le 19 décembre 1986. Accepté le 29 mars 1987.

\section{ADDENDUM}

Les travaux présentés dans l'article précédent de LACOINTE \& ZINsou (1987) ont été réalisés avec la collaboration technique de C. Constant (Laboratoire de Physiologie et Biochimie végétales) et du Personnel de la Station d'Agronomie du C.R.A.A.G.

\section{RÉFÉRENCES BIBLIOGRAPHIQUES}

Arnolin R., 1981. Quelques observations sur le cycle végétatif de Dioscorea alata, cv. "Tahiti » suivant la date de plantation. Communication, XVIIth Annual Meeting C.F.C.S., Caracas, 9 p.

Campbell J. S., Chukwueke V. O., Teriba F. A., Ho-A-Shu H. V. S., 1962. Some physiological investigations into the White Lisbon Yam (Dioscorea alata L.). II. Growth period and out-of-season production. Emp. J. exp. Agric., 30, 232-238.

Clairon M., Zinsou C., 1980. Etude de plantations échelonnées d'igname (Dioscorea alata, cv. « Lupias ») : effet du vieillissement du tubercule sur la croissance et le développement de la plante. In : "L'Igname", Séminaire Int., Pointe-à-Pitre, Guadeloupe, édit. I.N.R.A., Paris, 125-141.

Dagnélie P., 1975. Théorie et méthodes statistiques. Applications agronomiques. Vol. 2. Les méthodes de l'inférence statistique. Les Presses agronomiques de Gembloux, Vander, Bruxelles, $463 \mathrm{p}$.

Gooding E. G. B., Hoad R. M., 1967. Problems of yam cultivation in Barbados. Proc. Ist Int. Symp. Trop. Root Crops, Trinidad, 3, 137-151.
Jolivet E., 1969. Physiologie de la tubérisation (mise au point). Ann. Physiol. vég., 11, 265-301.

Lacointe A., Zinsou C., 1987. Croissance et développement au champ de l'igname (Dioscorea alata) à partir de plants produits par culture in vitro. Agronomie, 7 (5), 331-338.

Mathurin P., 1982. Nouvelle contribution à l'étude de la reproduction végétative de l'igname: sevrage et repiquage répétés du tubercule de Dioscorea alata, $c v$. « Pacala ». Thèse Doct. Ing., Université d'Abidjan, $126 \mathrm{p}$.

Miginiac E., 1980. Développement de quelques espèces d'ignames. In : "L'igname ", Séminaire Int., Pointe-à-Pitre, Guadeloupe, édit. I.N.R.A., Paris, 119-123.

Njoku E., 1963. The propagation of yams (Dioscorea spp.) by vine cuttings J. W. Agric. Sci. Ass., 8 (1), 29-32.

Perennec P., Madec P., 1980. Age physiologique du plant de pomme de terre. Incidence sur la germination et répercussions sur le comportement des plantes. Potato Res., 22, 305-317. 